\title{
Variations
}

Variations

Revue internationale de théorie critique

18 | 2013

"La poésie est une arme chargée de futur »

\section{La crise comme aubaine sans précédent pour le capitalisme : une nouvelle accumulation par la marchandisation des services publics}

\section{Ursula Huws}

Traducteur : José Chatroussat

\section{OpenEdition}

\section{Journals}

Édition électronique

URL : http://journals.openedition.org/variations/643

DOI : 10.4000/variations.643

ISSN : 1968-3960

Éditeur

Les amis de Variations

Référence électronique

Ursula Huws, «La crise comme aubaine sans précédent pour le capitalisme : une nouvelle

accumulation par la marchandisation des services publics », Variations [En ligne], 18 | 2013, mis en ligne le 31 mai 2013, consulté le 20 avril 2019. URL : http://journals.openedition.org/variations/643 ; DOI : 10.4000/variations.643

Ce document a été généré automatiquement le 20 avril 2019.

Les ami•e•s de Variations 


\title{
La crise comme aubaine sans précédent pour le capitalisme : une nouvelle accumulation par la marchandisation des services publics
}

\author{
Ursula Huws \\ Traduction : José Chatroussat
}

I

Cet essai soutient que 2008 marque un tournant pour le capital international dans la mesure où la crise financière lui fournit une occasion sans précédent de s'engager dans une nouvelle phase d'accumulation. Elle est fondée, non pas sur ce qu'on pourrait appeler " la première accumulation primitive » (la création de nouvelles marchandises à partir de ressources naturelles ou d'activités effectuées en dehors de l'économie monétaire) mais sur la marchandisation des services publics. Dans ce processus de marchandisation qui peut être considéré comme une sorte de "deuxième accumulation primitive", les activités déjà effectuées dans l'économie et rétribuées pour leur valeur d'usage (telles que l'éducation ou la santé) sont standardisées de telle sorte qu'elles peuvent être appropriées par le capital et gérées pour faire du profit. Ainsi la valeur d'usage est transformée en valeur d'échange. ${ }^{1}$ Cette seconde forme d'accumulation est basée sur l'expropriation, pas seulement de la nature, d'aspects non aliénés de la vie ou de tâches domestiques non payées, mais des résultats des luttes passées des travailleurs pour redistribuer de la plusvalue sous la forme de services publics universels. Elle constitue donc une réappropriation et, en tant que telle, ses impacts sur la vie de la classe ouvrière sont multiples et pernicieux. 
2 Pour les travailleurs accomplissant vraiment des services publics, de nouvelles formes d'aliénation sont introduites et il y a en général une détérioration des conditions de travail. Cependant, il y a aussi de grandes implications pour les travailleurs des autres secteurs parce que dans la plupart des économies développées, les travailleurs du secteur public restent le dernier bastion de la puissance syndicale et du maintien de conditions de travail décentes, définissant les normes auxquelles aspirent les autres travailleurs. Cela signifie que l'érosion des capacités de négociation des travailleurs des services publics représente aussi une défaite pour tous les travailleurs au niveau de leurs capacités en tant que travailleurs. À un niveau encore plus général, les avantages acquis dans le passé sont arrachés à l'ensemble de la classe ouvrière (y compris pour ses enfants, les personnes âgées, les malades et les chômeurs). Ces dernières conséquences ne peuvent pas, bien sûr, rester invisibles et deviennent tout naturellement des points centraux d'opposition. Cependant, une stratégie politique fondée seulement sur « la lutte contre les coupes dans les budgets sociaux » risque de donner l'impression que c'est simplement la taille des dépenses de l'Etat qui est en cause, rendant invisible la logique sous-jacente de marchandisation et cette réalité nouvelle : les services publics eux-mêmes sont devenus le terrain d'une accumulation qui est cruciale pour l'expansion permanente du capital international. Il s'agit d'une réalité nouvelle au sein de laquelle d'importantes sections du capital ont vraiment intérêt à investir dans un secteur du service public élargi, mais dans lequel les services sont standardisés et en mesure d'être assurés par une main-d'œuvre docile et interchangeable, intégrée dans une division mondiale du travail et soumise à la discipline de ce marché du travail mondial. Cela suscite de nouvelles contradictions dans les rapports entre l'Etat et le capital.

3 Il est de plus en plus difficile, pour ne pas dire impossible, de séparer le «capital financier » du " capital productif », que ce soit au niveau de l'analyse ou de l'expérience. ${ }^{2}$ Je ne tenterai pas ici de démêler les interactions complexes entre la période qui a précédé la crise financière et la restructuration des sociétés transnationales, ou les manières dont les compagnies en holding qui possèdent des sociétés soi-disant non-financières se comportent de plus en plus comme des groupes financiers. Néanmoins, afin de comprendre ce phénomène, il est nécessaire de mettre en relief certaines des conditions en arrière-plan qui ont conduit à l'émergence d'un nouveau type de firmes multinationales qui grossissent actuellement grâce à la marchandisation des services publics.

\section{II}

4 La crise financière de 2008 a coïncidé avec une crise de profitabilité pour le capital international qui avait déjà entrepris des restructurations massives. Un des aspects de cette restructuration a été la croissance énorme de la concentration du capital. 2007 a représenté un pic dans les flux des investissements mondiaux, avec des investissements mondiaux directs à l'étranger (FDI) atteignant leur plus haut niveau (1 833 billions de \$), surpassant le pic de l'an 2000. ${ }^{3}$ Un niveau record a également été atteint dans les fusions et acquisitions transfrontalières, leur nombre augmentant de $12 \%$ et leur valeur (quelque 1637 billions de \$) de plus de $21 \%$ par rapport à l'année précédente. ${ }^{4}$ L'UNCTAD a estimé que les ventes totales de 79000 sociétés transnationales et leurs 790000 filiales à l'étranger se montaient cette année-là à 31 trillions de $\$-21 \%$ d'augmentation depuis 2006 - tandis que le nombre total de leurs employés se montait à quelque 82 millions. Les 
cent plus grandes sociétés transnationales en particulier, ont renforcé leur emprise mondiale avec des actifs combinés à l'étranger estimés à 570 billions de $\$ .{ }^{5}$ Cependant, malgré cette croissance énorme, le nombre des projets écologiques d'investissements directs à l'étranger (FDI) a en fait diminué - de 12441 en 2006 à 11703 en 2007. ${ }^{6}$ Cela indique que, tandis que le processus de concentration s'est accéléré, il y a eu en fait un ralentissement dans la création de nouvelles productions. En d'autres termes, les plus grandes sociétés transnationales ont poursuivi leurs profits, non pas tant en raison de nouvelles productions qu'en cannibalisant les capacités de productions préexistantes. Sans une nouvelle source de marchandises à partir desquelles de la plus-value puisse être créée, les pré-conditions étaient en place pour un déclin de la profitabilité. Alors que très peu de parties du monde restaient hors du cadre du capitalisme mondial, où pouvait-on trouver ces nouvelles marchandises?

5 Cette tendance s'est trouvée associée à une réorganisation fondamentale majeure de la chaîne de valeur. Elle a été facilitée par une combinaison de politiques commerciales néolibérales et l'introduction généralisée des technologies de l'information et de la communication qui ont rendu facile la délocalisation des activités économiques et leur gestion à distance. La décennie précédente a ainsi vu une accélération de la tendance à moduler les processus des affaires d'une façon telle qu'ils ont pu être reconfigurés en une foule de permutations et de combinaisons contractuelles et spatiales différentes. À la fin des années 1990, «l'externalisation offshore » semblait encore une expérience risquée. ${ }^{7}$ Dix ans plus tard, cela apparaissait à tel point comme un volant normal de la gestion habituelle que les hommes d'affaires américains et européens étaient censés justifier pourquoi ils n'avaient pas ouvert des centres d'appel en Inde, partagé le travail de centres de service en Russie ou des studios de design au Vietnam, à côté de leurs équipements de production en Chine. La plupart des grandes sociétés ont systématiquement disséqué leurs processus de gestion en les décomposant en unités standardisées. Elles ont décidé, en les analysant une à une, si elles devaient les concentrer sur un seul site ou les répartir autour du monde, si elles devaient les garder à la maison-mère ou les délocaliser, si elles devaient rechercher le plus bas prix ou la plus haute qualité ou encore une combinaison complexe de ces différentes données. Au total, ces décisions ont entraîné des bouleversements considérables. En 2008 une nouvelle division du travail a émergé, ${ }^{8}$ avec de nouvelles structures de spécialisation régionale et de nouvelles configurations des secteurs et des entreprises. Comme de plus en plus d'activités économiques devenaient négociables, les grandes compagnies se lancèrent dans un double processus d'agrégation et de désagrégation, brassant et rebrassant ces activités dans de nouvelles combinaisons. ${ }^{9}$ Tandis que certaines entreprises continuaient à se centrer sur leurs capacités traditionnelles de fabrication ou d'extraction de ressources naturelles, d'autres consolidaient leurs positions comme prestataires de services. En 2006, une sur cinq (soit $20 \%$ ) des cent plus grandes sociétés transnationales non financières recensées par l'UNCTAD était une entreprise de services alors qu'elles n'étaient que 7\% en 1997.

6 A mesure que ces immenses entreprises de service développaient leurs marchés, les services qu'elles fournissaient devenaient plus génériques, prenant de plus en plus le caractère de marchandises normalisées, tellement normalisées que dans de nombreux cas, il leur était possible de fournir fondamentalement les mêmes services à des entreprises clientes (par exemple des services informatiques, l'administration du personnel ou des services à la clientèle), indépendamment du secteur économique où elles se trouvaient : fabrication, vente au détail, services publics ou d'autres secteurs. La 
plupart des entreprises clientes n'ont pas été longues, comme elles avaient pu l'être dans le passé, à intervenir sur un marché demandeur, en acquérant des services faits sur mesure, conçus en fonction de leurs besoins individuels. Bien mieux, elles sont devenues comme des gens faisant leurs courses dans une chaîne de magasins, faisant leur choix parmi une gamme de modèles de série proposés par les vendeurs. Dès qu'une telle offre atteint une masse critique, une logique économique rigoureuse se déclenche. Plus le marché de ces services s'élargit et plus cette standardisation peut être mise en œuvre. Ensuite le prix le plus bas peut être obtenu. Bientôt, même les clients qui préféraient continuer à effectuer des services pour eux-mêmes, sur place, ou bien les acheter sur mesure à un petit fournisseur local, sont guidés par la logique implacable du marché (sur lequel le coût relatif de la fabrication sur mesure est devenu exorbitant comparé à l'achat de produits standards). Ils sont amenés à réaliser qu'une telle personnalisation est un luxe et suivent la foule vers le fournisseur le moins cher. Dans les services d'affaires fondés sur l'informatique en particulier, cette logique leur a donné un dynamisme supplémentaire grâce à la prédominance d'ensembles de logiciels standards (comme ceux fournis par Microsoft) ou de plates-formes (comme celles fournies par SAP Business Management Systems) et des moyens par lesquels ils peuvent être mis en réseaux avec les services fournis par des fournisseurs mondiaux en télécommunications, en énergie ou en d'autres services d'infrastructure. Cependant, les industries activées par l'informatique ne sont en aucune manière les seuls exemples de marchés vendeurs dans le domaine de la prestation de services. De grandes sociétés multinationales s'engagent également de plus en plus dans la prestation de travail manuel, que ce soit par la sous-traitance de maind'œuvre par des agences de travail temporaires ou par la prestation de services extérieurs de sécurité, de soins ou de nettoyage par exemple. Au cours de la dernière décennie, les administrations du secteur public ont fait partie des clients prépondérants ayant recours à ces entreprises de services.

7 Dans les premières années du vingt-et-unième siècle, ces tendances se sont mutuellement renforcées, engendrant une situation dans laquelle les grandes entreprises prestataires de services (avec leur propre division mondiale interne du travail) cherchaient à tout prix à s'étendre. Etant donné les occasions restreintes de croissance par fusions ou acquisitions et les marchés étant proches de la saturation dans de nombreux secteurs, le secteur public offrait un nouveau champ d'expansion attractif. En 2008, selon un rapport publié par le gouvernement britannique, les services publics sous-traités représentaient $6 \% \mathrm{du}$ PNB du Royaume-Uni, employant plus de 1,2 million de personnes, avec un chiffre d'affaires de 79 milliards de $€$ en 2007-2008, soit une augmentation de 126\% par rapport aux 31 milliards estimés en 1995-96. Le rapport baptisait ce secteur en rapide expansion 'the public services industry' (PSI) et relevait qu'en termes de valeur ajoutée, il était « de façon significative plus important que ceux de l'alimentation, des boissons et du tabac ( 23 milliards de $€$ ), des communications ( 28 milliards de $€$ ), de la fourniture d'électricité, du gaz et de l'eau (32 milliards de $€$ ) et que celui des hôtels et de la restauration (36 milliards de $€$ )». Ce phénomène n'est pas particulier à la Grande-Bretagne. La part du PNB du secteur de l'industrie des services publics était estimée à un niveau plus élevé en Suède et en Australie. En chiffres absolus, ce marché au Royaume-Uni, avec ses 79,4 milliards, arrivait en deuxième place derrière celui des Etats-Unis (394 milliards). Mais il était néanmoins d'une ampleur significative comparée à sa valeur estimée à 44,8 milliards en France, à 32,2 milliards en Australie et à 24,7 milliards en Espagne. Si on devait appliquer une définition plus large englobant les anciens services publics comme la poste, les 
télécommunications, l'eau et l'énergie, ces chiffres seraient considérablement plus élevés. 10

8 Aussi élevées que soient ces sommes, elles ne représentent qu'une fraction du total de la valeur des services publics. En dépit de la rhétorique néolibérale contre le Big Government ( l'Etat pléthorique ») depuis un quart de siècle, malgré les coupes claires dans les services qui ont été vécues par les travailleurs comme un retrait du soutien de l'Etat et malgré la vente d'actifs publics, ${ }^{11}$ les dépenses étatiques ont augmenté inexorablement dans tous les pays de l'OCDE, en chiffres absolus comme en pourcentage du PNB. Tandis qu'en 1960 ces dépenses tournaient autour de 28,4\% du PNB dans l'ensemble de l'OCDE, en 1980 elles avaient grimpé à $43,8 \%$ pour continuer à bondir et atteindre $47,7 \%$ en 2009 . Cela varie quelque peu selon les pays. Les dépenses de l'Etat représentent 39,7\% au Japon, $42,2 \%$ aux Etats-Unis et $43,8 \%$ au Canada, les situant vers les derniers rangs de la liste tandis que les Pays-Bas, la Suède, la France, l'Autriche, la Belgique et l'Italie se trouvant dans les premiers rangs avec des dépenses étatiques entre $50 \%$ et $54 \%$ du PNB. Le Royaume-Uni et l'Allemagne sont proches de la moyenne avec respectivement $47,2 \%$ et $47,6 \%{ }^{12}$ Une analyse fondée sur la dépense de l'Etat par habitant entrâne un classement différent, les Etats-Unis (ou le total des dépenses gouvernementales est estimé à presque 6 trillions de \$) devant l'Italie, le Canada, la Grande-Bretagne et le Japon, en partie à cause de leurs dépenses militaires beaucoup plus élevées..$^{13}$ Quelle que soit la façon d'analyser les chiffres, cela représente un potentiel d'expansion qui prend des dimensions stupéfiantes. Et il est assez ironique de constater que ce marché, à l'exception notable des Etats-Unis particulièrement militarisés, est proportionnellement plus important précisément dans les pays qui, sous la pression démocratique venant d'en bas, ont construit des Etats offrant la protection sociale la plus étendue. C'est pour cette raison que la Suède sociale-démocrate considérée par Esping-Andersen ${ }^{14}$ et d'autres comme ayant atteint le plus haut degré de nationalisation parmi toutes les économies capitalistes développées, a trouvé le moyen d'être en tête de la liste des pays ayant la part la plus importante de services publics sous-traités en 2008. En somme, plus le secteur nonmarchand est important, plus le champ pour la marchandisation est étendu.

III

Comment ce marché est-il devenu accessible? L'obtention de services par des fournisseurs extérieurs n'est pas nouvelle, bien sûr. Pendant des siècles, les Etats ont commissionné des fournisseurs privés pour la construction de bâtiments, de routes, de ponts et autres « travaux publics » ainsi que pour l'achat de biens allant des trombones de bureaux aux engins contre les incendies. Et au cours de toutes les époques, il y a toujours eu des scandales à propos des pots-de-vin versés à des fonctionnaires pour obtenir ces contrats lucratifs. Il est cependant peut-être plus utile de dater la vague actuelle de soustraitance au début des années 1980. Au Royaume-Uni, le gouvernement conservateur a lancé deux formes différentes de privatisation caractéristiques. L'une d'elles a été la vente directe d'actifs publics présentée à l'origine comme des ventes à des citoyens individuels plutôt qu'à des entreprises. Parmi ces ventes, les plus profitables ont été les ventes de logements municipaux à leurs locataires et à partir de 1984, la vente de services publics (télécommunications, gaz et électricité) sous forme d'émission d'actions que le public était invité à acheter, avec à l'appui une vaste campagne publicitaire. En association avec ces ventes mais avec beaucoup moins de tapage publicitaire, il y eut l'ouverture à la 
concurrence pour les entreprises privées des marchés de l'énergie et des télécommunications. L'autre forme de privatisation (n'impliquant pas un changement complet de propriétaire) a été l'introduction en force par le gouvernement des « appels d'offres compétitives obligatoires ", d'abord dans les administrations locales et ensuite dans le Service National de Santé (NHS). Bien que cela ne signifiât pas nécessairement que les services concernés devaient être accomplis par des entrepreneurs extérieurs, les services internes employant des fonctionnaires étaient à présent obligés d'être en concurrence avec les entreprises privées afin d'être en mesure de continuer à effectuer les services en question. Cela entraîna une pression à la baisse sur les salaires et les conditions de travail et introduisit une nouvelle précarité. Les emplois ne furent plus garantis « à vie » mais seulement pour la durée d'un contrat.

Cette première enveloppe d'appels d'offres concernait principalement des tâches telles que celles du bâtiment, du nettoyage, du recyclage des ordures et, de façon peut-être bien non fortuite, des domaines où les syndicats du secteur public étaient puissants et où ils ont montré leur force au travers de grandes grèves lors de "l'hiver du mécontentement » de 1978-79 qui avait immédiatement précédé l'élection victorieuse de Thatcher. Une bonne partie de la rhétorique entourant le recours à la sous-traitance n'insistait pas seulement sur la supposée efficacité qu'on allait gagner en confiant des services à des entreprises privées, non bridées par les "pratiques restrictives» du secteur public manuel syndicalisé ; cette rhétorique était aussi centrée sur un discours d'entreprise. On prétendait ainsi que la prestation externe de ces services créerait des débouchés pour de nouvelles petites entreprises. En réalité la majorité des contrats allèrent à de grands groupes et souvent à des multinationales. En 1984-85 par exemple, au moment où l'attention du public était focalisée sur la grève nationale des mineurs - l'autre groupe de travailleurs organisés ciblé par le gouvernement conservateur de Thatcher - une autre grève de longue durée s'engageait à l'hôpital Barking dans l'Est de Londres. Les nettoyeurs en grève dans cet hôpital étaient employés par une filiale du Pritchards Services Group, une firme transnationale ayant 58 filiales dans 15 pays, employant 17000 personnes dans 430 hôpitaux dans le monde, y compris en Arabie saoudite, en Afrique du Sud, en Nouvelle-Zélande, en France, en Allemagne et aux Etats-Unis. ${ }^{15}$ Il est intéressant de relever que de 1983 à 1994, le mari de Thatcher, Denis, a été le vice-président de Attwoods Société anonyme, une grande firme internationale d'organisation du nettoyage qui devait précisément profiter de cette forme de privatisation. Une étude détaillée sur l'impact des «appels d'offres compétitives obligatoires » appliqués aux administrations locales au Royaume-Uni en 1993-94 concluait que les femmes ont été beaucoup plus touchées défavorablement que les hommes. Les emplois féminins dans les administrations locales ont baissé de $22 \%$ contre $12 \%$ pour les emplois masculins, de même que les revenus des femmes ont davantage dégringolés que ceux des hommes. Il y a eu également une chute substantielle du nombre des syndiqués. Pour autant, les implications pour l'Etat en termes de dépenses ont été en fait négatives. Tandis que les propagandistes néolibéraux (au sein de la Banque Mondiale, du FMI et de l'OCDE aussi bien que du gouvernement britannique) prétendaient que cette politique permettait d'économiser 20 à $25 \%$ des dépenses, en réalité les économies ne s'élevèrent en moyenne qu'à 6,5\%. Dans les 39 autorités administratives étudiées, leur montant est estimé à 16 millions de $€$. Cependant les coûts publics totaux (en prenant en compte la perte des contributions de l'assurance nationale et le coût des allocations chômage afférentes) étaient estimés à 41 millions de $€$ (dont 32 millions alloués à l'emploi des femmes). En 
extrapolant au niveau national, on a estimé que les économies s'élevaient à 124 millions et les pertes à 250 millions, soit une perte nette de 126 millions de $€ .{ }^{16}$

11 Chacune de ces formes de privatisation a des équivalents ailleurs. En Europe, la GrandeBretagne a joué un rôle important en impulsant un programme de libéralisation qui a conduit à une liquidation forcée tout d'abord des prestataires des télécommunications nationales, puis des entreprises publiques énergétiques et ensuite à l'ouverture des services postaux au marché. Il y avait depuis 1966 une réglementation par l'Union Européenne concernant les appels d'offres publiques: la directive 66/683 interdisait les dispositions favorisant les prestataires nationaux aux dépens des prestataires étrangers à l'intérieur du Marché européen. Le tournant du néolibéralisme a introduit une plus grande déréglementation au milieu des années 1980. L’Acte unique européen en 1986 a introduit un nouveau régime selon lequel les procédures d'adjudication sont mises en place comme normes dans tous les services publics au sein de l'Union Européenne et selon lequel des procédures négociées ne sont autorisées que dans des circonstances exceptionnelles. La première Directive des secteurs de base (90/351) a supprimé les barrières d'accès au marché de l'énergie, des télécommunications, des transports et de l'eau. Et en 1992 la Directive des services (92/50) a étendu les principes qui régissaient les appels d'offres concernant les biens, les travaux publics et les secteurs de base à l'ensemble des services publics. ${ }^{17}$

12 Pendant ce temps, l'Uruguay Round du GATT (accord général sur les tarifs et le commerce) qui a commencé en 1986 pour aboutir au GATT de 1994, plaça les services (de même que le capital et la propriété intellectuelle) dans le cadre des accords commerciaux mondiaux. L'année 1992 a vu la mise en place d'une agence des Nations Unies, l'International Telecommunications Union (ITU), et a été le début d'une époque de dérégulation mondiale des télécommunications qui a offert à son tour l'infrastructure permettant à l'échelle mondiale le transfert à bas prix de données numériques. Ce fut aussi l'année où l'Inde a été capable de commencer à exporter en abondance ses services informatiques grâce à la suppression des barrières douanières qui avaient été conçues à l'origine pour protéger l'industrie locale comme élément d'une stratégie de substitution des importations. Au début des années 1990, la scène était donc mise en place pour que les firmes mondiales puissent fournir une série de services en passant par-dessus les frontières nationales, se frayant une voie dégagée de toutes les mesures de restriction qui avaient pu être mise en place pour protéger les entreprises nationales ou les mainsd'œuvre locales. ${ }^{18}$

13 Ces développements ont coïncidé historiquement avec la fin officielle de la Guerre froide après 1989. Cela n'a pas seulement permis d'ouvrir de nouveaux marchés au capital occidental dans les pays de l'ancien bloc soviétique mais aussi d'écarter les dernières raisons pouvant subsister chez les employeurs, pour démanteler, en collaboration avec les gouvernements nationaux, les accords spéciaux avec les organisations du mouvement ouvrier qui avaient caractérisé le troisième quart du vingtième siècle (diversement qualifié de «fordisme », d' "âge d'or ", d'« Etat providence keynésien de l'après-guerre », etc.). ${ }^{19}$

\section{IV}

L'érosion de ces accords spéciaux fait partie du contexte de développement d'une nouvelle division mondiale du travail mise en œuvre par le nouveau secteur des 
multinationales dédié aux services. Que ces firmes réalisent leurs économies d'échelle en faisant faire le travail à des endroits où les compétences sont abondantes et bon marché (" délocalisation ») ou en faisant venir de la main-d'œuvre bon marché sur les sites où s'effectue le travail, par exemple le recours à des travailleurs immigrés, elles adoptent en fait la même stratégie en puisant dans l'armée de réserve mondiale du travail. Bien sûr, leurs choix ne sont pas exempts de contraintes. Elles doivent manœuvrer à l'intérieur de limites, par exemple l'apport en main-d'œuvre adaptée et qualifiée, les règlements nationaux qui restreignent les mouvements des travailleurs, établissent un salaire minimum ou imposent des normes de qualité particulières. Une autre contrainte est de savoir dans quelle mesure la main-d'œuvre à laquelle elles ont à faire est capable d'engager une lutte. Toutefois, dans la mesure où les employeurs peuvent puiser dans des sources de travail alternatives, cela constitue une menace pour les conditions de travail et le niveau des salaires des travailleurs et cela agit sur elles et sur eux comme une force disciplinaire.

L'existence de cette nouvelle armée de réserve mondiale semble directement liée à un lent processus d'effilochage des normes de travail au sein de la partie privilégiée de la main-d'œuvre du capitalisme, avec ses perspectives de sécurité de l'emploi, de promotion, ses régimes de retraite auxquels contribuent les employeurs, ses congés payés, ses allocations maladie, ses droits à la maternité et les autres acquis obtenus au cours du troisième tiers du vingtième siècle. Bien sûr, cette évolution ne peut pas être entièrement attribuée à la mondialisation du travail. Les attaques directes contre les travailleurs organisés, sous Thatcher et Reagan par exemple, ont aussi clairement joué un rôle majeur. Cependant, l'existence d'une armée de réserve mondiale du travail est certainement le facteur majeur pour expliquer l'échec des travailleurs, y compris des travailleurs qui historiquement étaient très qualifiés et bien organisés, à résister à la détérioration de leurs salaires et de leurs conditions de travail aux cours des deux dernières décennies. Cette détérioration est clairement mesurable en termes d'horaires de travail plus longs, de dégradation de la santé physique et mentale, de la baisse du pouvoir d'achat des salaires, de la perte de la couverture des retraites et de la permanence des contrats d'embauche. Il est difficile d'échapper à la conclusion que la position pour négocier des travailleurs avec leurs employeurs a été sérieusement minée $\mathrm{du}$ fait qu'on sait que d'autres travailleurs sont tout à fait capables ailleurs d'accomplir leurs jobs.

Il faut relever que dans la plupart des pays développés jusqu'en 2008, l'exception majeure à cette tendance à l'érosion se trouvait parmi les travailleurs du secteur public. Au cours des deux dernières décennies, dans de nombreux pays, les travailleurs du secteur public n'étaient pas seulement devenus la partie de la main-d'œuvre la plus syndiquée ${ }^{20}$ mais aussi celle où perduraient encore les carrières constituant des modèles de ce à quoi peut ressembler un travail décent. Les travailleurs du secteur public étaient en pointe, par exemple pour négocier des accords donnant des chances égales, des compromis entre temps et argent qui favorisaient un meilleur équilibre entre vie et travail et le renforcement des normes qui plaçaient la qualité des services aux clients au-dessus des considérations financières.

17 Ce n'est pas ici l'endroit pour discuter si on peut dire qu'un "modèle fordiste " unique dans les relations industrielles de l'après-guerre a existé. Quels qu'aient été les compromis particuliers conclus entre le capital et le travail dans les différents contextes nationaux, et quel que soit le degré déjà atteint dans leur démantèlement par les 
politiques néolibérales entamées au cours des années 1980, on peut affirmer avec certitude que 1989 marque un moment où les pressions ont commencé pour évoluer vers un modèle convergent global de l'emploi, presque partout dans le monde; et cela pour exercer une plus grande force visant à contrecarrer les pressions émanant du monde du travail afin de protéger et étendre ses acquis antérieurs.

Ce processus n'a bien sûr pas été entièrement négatif pour tous les travailleurs. À cause des énormes disparités entre pays et la nature très segmentée des marchés du travail aussi bien que les degrés très différents auxquels les travailleurs ont été capables de résister, ces processus de nivellement ont représenté une amélioration relative pour certains; même si en même temps, ces processus se sont traduits pour d'autres par une détérioration des salaires et des conditions de travail. En particulier des femmes et des gens de différentes minorités ethniques noires et autres, des travailleurs à temps partiel et des travailleurs des pays ayant une histoire très faible en matière de protection de l'emploi, bénéficièrent au cours des années 1990 de différentes directives européennes ou de l'Organisation Internationale du Travail contre les discriminations. Néanmoins une part importante du contexte d'ouverture des services publics au marché a été l'érosion simultanée de la protection de l'emploi et l'accès facilité à un pool mondial de travail: une armée de réserve pas seulement de travailleurs manuels de "travailleurs de l'information » qui, grâce à la standardisation du travail des cols blancs par le biais de l'introduction de technologies de l'information et de la communication, sont de plus en plus capables d'accomplir des tâches qui étaient auparavant une composante de celles des fonctionnaires et des employés de bureau du secteur public. La standardisation des tâches et la nature générique croissante des processus de travail accomplis par les cols blancs (combinés avec la facilité avec laquelle les données informatiques peuvent être transmises à distance) ont rendu de façon nouvelle les employés de bureau substituables les uns aux autres, sapant leur pouvoir de négociation avec les employeurs que ce soit dans le public ou dans le privé.

\section{V}

Loin de modifier les effets les plus pernicieux pour les travailleurs de la marchandisation des services publics des années 1980 au début des années 1990, le gouvernement du New Labour élu au Royaume-Uni en 1997 a poursuivi agressivement la privatisation. Dans les administrations locales par exemple, il a remplacé ce que les conservateurs avaient appelé « Compulsory Competitive Tendering » (appel d'offres compétitive obligatoire) par sa propre initiative appelée «Best Value » (la meilleure valeur). Celle-ci a mis en place l'obligation légale pour les conseils locaux de garantir les services les plus économiques, efficients et efficaces; ils devaient démontrer qu'ils avaient comparé tous leurs services avec ceux des autres prestataires de services publics et privés. Cela a également introduit un régime de contrôle et d'audit permanents réduisant le domaine d'intervention du professionnalisme individuel et la capacité des travailleurs à répondre directement aux besoins des clients. Tandis qu'il y avait moins d'obligations légales immédiates apparentes pour la sous-traitance, le champ des obligations légales générales s'est élargi (ainsi que certaines sanctions en cas d'incapacité à démontrer la "Best Value ", juste dissuasives si ce n'est davantage). Mais le plus important est peut-être ceci. L'introduction de cette politique a entraîné un changement de mentalité chez les responsables locaux, quelle que soit leur appartenance politique, en les amenant à intérioriser des valeurs imposées par le 
système. Même si les services n'étaient pas sous-traités, ils devaient faire comme s'ils l'étaient, avec des fonctionnaires de plus en plus soumis à la discipline du marché. La condition première pour faire les comparaisons requises était que les services soient définis selon des modalités standardisées. "Best Value » peut être ainsi considérée comme un des moteurs du processus pour rendre les tâches routinières et standardisées. Cela s'est accompagné de l'introduction d'indicateurs de performance et de protocoles permettant de vérifier les tâches statistiquement ainsi que de fournir les bases de normes de qualité inscrites dans les contrats ou les Service Level Agreements (SLAs) qui définissent les termes dans lesquels les entreprises fournissent ce qu'étaient autrefois les services publics.

Depuis que la standardisation est un prérequis de la marchandisation, une telle politique du New Labour a donc joué un rôle crucial dans la marchandisation des services publics qui sous-tend leur transformation en unités d'échange sur le marché mondial. ${ }^{21}$ Les collectivités locales n'ont pas été la seule cible. Le New Labour a aussi introduit des réformes majeures qui ont développé un marché pour les entreprises privées Service de santé national (NHS), ${ }^{22}$ l'éducation, les prisons et la justice. ${ }^{23}$ Dans chaque cas, le processus de transformation d'une partie du service public en une marchandise vendable passe par les mêmes étapes: standardisation, création d'une demande, action pour persuader le personnel d'accepter les changements et le transfert des risques. ${ }^{24}$ Bien évidemment ces évolutions n'ont pas été le fait uniquement du New Labour, ou du Royaume-Uni. Cependant, leur adoption enthousiaste par les sociaux-démocrates en Grande-Bretagne comme en Scandinavie et ailleurs a joué un rôle important dans la création d'un nouveau "bon sens » selon lequel il apparaît à la fois naturel et inévitable que les normes soient définies par le marché.

21 Dans le cas des services personnels complexes (comme l'enseignement, la santé ou le travail social) exigeant une masse de connaissances tacites et contextuelles, une compétence en communication et en "travail émotionnel ", ${ }^{25}$ les processus de standardisation, qui sous-tendent la marchandisation, ne sont pas toujours faciles à mettre en œuvre. Ils impliquent plusieurs étapes au cours desquelles la connaissance tacite est progressivement codifiée, les tâches sont standardisées, les mesures de rendement sont acceptées, les procédures de management sont réorganisées, les organismes sont brisés en leurs éléments constituants. Ces éléments sont formalisés, parfois comme des entités légales séparées, et des relations de type marchand sont introduites entre elles. Tout cela peut très bien être le préambule à un changement de propriétaire ou à l'ouverture à un acquéreur extérieur. Ce n'est que lorsque l'activité a été de fait ou potentiellement transformée en quelque chose qui peut être fait ou vendu à une entreprise faisant du profit que le terrain est préparé pour une restructuration ultérieure par des voies qui constituent les pratiques normales des firmes multinationales : fusions, acquisitions, reconfiguration des parties en de nouvelles combinaisons et introduction d'une division mondiale du travail. ${ }^{26}$

La décennie de 1997 à 2007 a vu ces processus de standardisation et d'internationalisation se poursuivre rapidement. En 2000 un nouvel ensemble de protocoles et de normes mondiales de qualité a été déployé et mis en place. Il comprend les normes de qualité ISO. ${ }^{27}$ À cet égard les configurations requises pour les systèmes de gestion de la qualité ont grimpé de 46571 en 1993 à 1064785 en 2009, soit une augmentation stupéfiante de près de 13 fois plus en seize ans. ${ }^{28}$ La croissance mondiale des critères environnementaux a été également spectaculaire - de 13994 en 1999 à 223149 une décennie plus tard, soit 16 fois 
plus en dix ans. Entre temps des millions de travailleurs dans le monde ont obtenu des certificats validés par Sun, Oracle, Cisco, Microsoft ou d'autres firmes d'informatique, leur permettant d'accéder à un marché de l'emploi mondial et transparent de telle sorte que leurs compétences sont interchangeables avec celles d'autres travailleurs et parfaitement comprises par les employeurs. Il est par exemple possible pour un détenteur nouvellement qualifié d'un certificat par Cisco d'aller sur un site internet ${ }^{29}$ et de découvrir que cela lui fera gagner un salaire de 69401 \$ US au Royaume-Uni ou un salaire d'environ 14518 \$ US en Inde. Ceux qui ne peuvent pas vendre leurs qualifications à de grandes sociétés multinationales, peuvent les vendre aux enchères au plus offrant sur des sites comme oDesk. ${ }^{30}$ Pour donner une idée de la taille de l'armée de réserve mondiale créée de cette façon, il suffit de relever qu'une certification de Microsoft, le Microsoft Certified Professional (MCP) était détenu par 2296561 travailleurs au moment de la rédaction de cet essai ${ }^{31} \mathrm{Ce}$ nombre est stupéfiant pour un métier qui, pas plus tard que dans les années 1980, relevait du domaine réservé d'une petite élite ayant un pouvoir considérable pour négocier. ${ }^{32}$

\section{VI}

23 Nombre de ces sociétés pour lesquelles les travailleurs en cols blancs sont en concurrence pour un emploi, ont grandi de façon exponentielle au cours des vingt-cinq dernières années. Elles ont parfois leur origine dans le secteur de services de firmes manufacturières mais parfois avec des racines dans des firmes financières ou de services de gestion. Elles comprennent Siemens Business Services, Accenture, Capgemini et Capita, avec une base historique en Europe ou en Amérique du Nord. Mais elles comprennent aussi Infosys, Wipro and Tata Software Consultancy, des sociétés originaires d'Inde fournissant au début des services informatiques de bas niveau mais s'avérant bientôt capables d'évoluer rapidement dans la chaîne de valeur pour devenir des leaders mondiaux en fournissant la sous-traitance délocalisée d'opérations de gestion (Business Process Outsourcing). Il y a aussi des sociétés qui se centrent davantage sur l'approvisionnement de travailleurs manuels, comme Manpower et Group 4 Securicor, et des sociétés qui se spécialisent dans certains secteurs ou types de services (comme Vertex qui sous-traite des services de centres d'appels) aussi bien que d'autres qui embrassent de nombreux types d'activités, comme Serco et ISS.

Ces sociétés ne sont pas des acteurs passifs dans l'économie mondiale. Elles commercialisent activement leurs services aux gouvernements et pratiquent un vigoureux lobbying pour développer la sous-traitance, que ce soit individuellement ou en passant par des sociétés de gestion telles que la Business Services Association (BSA) et la National Outsourcing Association (NOA) au Royaume-Uni. Beaucoup ont des divisions de consultance qui expliquent aux corps de l'Etat comment "moderniser » leurs services, qui recommandent d'une main les différentes stratégies de sous-traitance dont de l'autre main, elles et d'autres sociétés sont les bénéficiaires. Ces consultants sont particulièrement actifs sur des marchés émergents tel que le Vietnam,,$^{33}$ où la grande taille des services de l'Etat leur offre des morceaux de premier choix. La société Serco a même mis en place un « Serco Institute » qu'elle décrit elle-même comme " un think tank britannique offrant une recherche et un leadership intellectuel dans l'utilisation de la concurrence et s'engageant dans la réforme des services publics et le développement de marchés pour des services publics durables. ${ }^{34}$ Leys et Player ont disséqué le lobbying 
extraordinairement agressif auprès du New Labour et des gouvernements de coalition au Royaume-Uni qui a façonné les propositions 2011 pour la réforme du Service National de Santé (NHS). ${ }^{35}$

De plus en plus, leurs rapports marchands avec les gouvernements qui sont leurs clients se transforment; les acheteurs détenant le pouvoir deviennent des vendeurs sur le marché. Un des facteurs contribuant à ce déplacement du pouvoir est le changement de propriété et le changement de nature de la qualification et des connaissances de la maind'œuvre. Traditionnellement de nombreux travailleurs des services publics (y compris des enseignants, des hospitaliers et des travailleurs sociaux) apportaient tout un ensemble complexe de compétences dans leur travail et étaient capables d'exercer leur métier avec un degré d'autonomie leur permettant de répondre de façon individualisée à leurs clients. Même dans des cadres dirigés de manière très bureaucratique, comme les services des impôts, ${ }^{36}$ de nombreux travailleurs du public avaient acquis une somme considérable de connaissances spécialisées découlant de leur expérience et pour la plupart non consignées par écrit. La qualité des services a par conséquent dépendu essentiellement de l'existence d'une main-d'œuvre stable, dévouée, qui souvent obéissait fortement à des critères éthiques et professionnels développés au travers de pratiques communes, avec un haut niveau d'autocontrôle.

Les processus qui constituent les conditions préalables à la marchandisation exigent une analyse de ces compétences et des tâches qui leur sont associées afin de les briser en leurs différentes composantes, d'établir des normes explicites pour leurs performances et souvent, d'introduire une division du travail élaborée permettant de transférer les besognes les plus routinières à des travailleurs moins qualifiés. Les formes de contrôle intériorisées par les travailleurs eux-mêmes et vérifiées aussi bien grâce à leur propre motivation que par les interventions en retour de leurs collègues ou de leurs chefs sont dès lors remplacées par des formes dictées de l'extérieur. Ce processus prend du temps et demande souvent la transmission graduelle du travail à une nouvelle cohorte de travailleurs ayant reçu une formation différente.

D'ailleurs, ce n'est pas seulement la main-d'œuvre qui doit être recyclée. Les usagers doivent également s'habituer à être traités comme des consommateurs sur un marché de masse au lieu d'être des clients individuels. Dans les premières étapes de la soustraitance, ce processus ne peut en aucune manière être total. Des considérations à la fois d'opportunité et d'efficacité exigent un transfert calme et en douceur qui est vécu par les usagers, dans les premières étapes, comme n'entraînant aucune détérioration et même, si possible, comme une amélioration à certains égards en comparaison avec le processus antérieur "qui gaspillait » et " était bureaucratique ». La façon la plus facile de procéder à une telle transition consiste à employer le même personnel pour offrir le même service. Ainsi, un premier type de sous-traitance d'un service donné (tout à fait comme dans le cas typique du rachat d'une entreprise) n'entraîne pas des licenciements massifs mais plutôt un transfert de personnel d'un employeur à un autre. En Europe, cela est facilité par la TUPE, la directive sur le Transfert des Entreprises (Protection de l'emploi) de l'Union Européenne, (directive du Conseil européen 2001/23). Elle apporte une protection légale aux employés transférés, avec le respect de leurs conditions de travail, y compris leurs prestations de pensions. L'existence de la TUPE a conduit à une situation où la réaction des syndicats à la sous-traitance consiste souvent à ne pas y résister directement mais à s'assurer avant tout que les employés transférés sont pleinement protégés par la réglementation de la TUPE. L'action syndicale est comparativement rare face aux 
problèmes liés à la sous-traitance dans le secteur public, même si elle n'est pas du tout inexistante..$^{37}$ Comme conséquence des transferts successifs sur les sites où la soustraitance a été mise en place en premier, la main-d'œuvre qualifiée des multinationales a augmenté, non pas tant par un nouveau recrutement sur le marché du travail que par des transferts. Dans un département fournissant des services informatiques par exemple ou dans un grand centre d'appels externalisé se situant l'un à côté de l'autre et avec des caractéristiques et des conditions d'emploi très différentes, héritées des employeurs précédents, on peut trouver des travailleurs qui ont occupé des postes antérieurement dans différents bureaux d'administrations centrales ou locales ou dans des banques, des entreprises manufacturières ou de services. Dès qu'ils sont employés par le sous-traitant, ils peuvent découvrir que leur travail subit une série de changements. Certaines tâches étant transférées vers d'autres branches de l'entreprise dans d'autres régions ou d'autres pays, pour celles restantes, ils peuvent avoir à affronter de nouveaux indices de performance ou de nouveaux objectifs, de nouvelles exigences pour être disponibles à des heures tenues auparavant comme inacceptables pour travailler. Depuis que les contrats de sous-traitance sont en général plutôt de courte durée, chaque renouvellement de contrat va entraîner encore plus de restructuration.

Dans une étude de cas au Royaume-Uni, le département informatique d'une administration locale a été sous-traité en premier à une grande firme mondiale d'informatique basée en Europe (Société A). Quelques employés ont été licenciés tandis que d'autres ont transféré leurs emplois vers cette société. Après quelques années, le contrat a dû être renouvelé et a été obtenu par une entreprise plus petite, établie au Royaume-Uni (Société B). Certains des employés transférés initialement sont restés dans la Société $\mathrm{A}$, réintégrés à des fonctions différentes, certains ont été licenciés et d'autres ont été embauchés par la Société B. Cette Société B a ensuite été rachetée par une multinationale établie aux Etats-Unis avec une forte tradition antisyndicale (Société C). La main-d'œuvre restante (qui continue à fournir les mêmes services informatiques aux mêmes autorités locales) a ainsi été employée par quatre employeurs différents en moins d'une décennie. Bien qu'aucune brèche formelle ne soit apparue dans le règlement du TUPE, il y a eu pour les employés une constante dégradation de leurs conditions de travail et de leur qualité de vie au cours de cette période. Un travailleur dont l'enfant était en train de mourir d'un cancer, avait demandé la permission au nouvel encadrement de la Société $\mathrm{C}$ de travailler à son domicile deux jours par semaine (quelque chose qui aurait été normal avec la convention collective de l'ancienne autorité locale). Il s'est vu refuser cette permission par un cadre qui lui a dit : «Si le travail peut être fait à domicile, il peut être fait en Inde. »

Un changement encore plus pénible pour les travailleurs du secteur public intervient: c'est le passage des valeurs de l'éthique du service public où le travail est ressenti comme ayant une signification intrinsèque, à un environnement commercial où le travail consiste uniquement à "remplir les poches des actionnaires » selon l'expression d'un technicien en informatique. De nombreux travailleurs avaient consciemment fait le choix de travailler dans le secteur public, sacrifiant les possibilités de promotion pour ce qui était considéré comme un travail sûr et gratifiant, apportant quelque chose à la collectivité. ${ }^{38}$ Ils peuvent bien devenir des travailleurs mécontents, refusant d'acquérir le comportement "réservé et dynamique » qui inspire le respect envers les entreprises multinationales pour lesquelles ils travaillent désormais, cela importe peu. Dès que leur compétence a été acquise par l'entreprise, ils peuvent être remplacés par une main- 
d'œuvre plus jeune, plus malléable, reconnaissante quelle que soit la sécurité qu'elle peut obtenir. Au Royaume-Uni, le gouvernement travailliste avait assuré une certaine protection aux employés de seconde génération dans les services publics sous-traités, qui garantissait que les nouveaux employés qui travaillaient aux côtés des travailleurs de l'ancien service public reçoivent la même paye et les mêmes pensions. Cependant ce code a été annulé en décembre 2010 par le nouveau gouvernement de coalition. ${ }^{39}$ En examinant les données du Labour Force Survey, une comparaison entre les conditions de travail aux mêmes postes dans les secteurs public, privé et bénévole au Royaume-Uni montre que dans tous les cas, les conditions sont pires dans le secteur privé. Par exemple, seulement $3 \%$ des gardiens de prison dans le secteur public ont moins d'un an de titularisation, contre $11 \%$ dans le secteur privé; $10 \%$ des travailleurs à plein temps qui soignent ou procurent des services à la personne dans le secteur privé, travaillent plus de 48 heures par semaine, contre $2 \%$ dans le secteur public. ${ }^{40}$

Dès que les connaissances des travailleurs de l'ancien secteur public ont été dépouillées, codées et placées dans des bases de données standard, elles peuvent être transférées vers des employés payés moins cher, mais être utilisées comme un atout par le nouvel employeur. Par exemple, une entreprise qui a déjà acquis l'expérience de faire fonctionner l'aide en ligne d'une administration locale, le système informatisé des Ressources Humaines d'une université, de fournir le fonctionnement informatique d'un service des impôts ou de s'occuper du service de lingerie d'un hôpital, est alors capable de commercialiser ces services agressivement à d'autres clients publics potentiels, dans d'autres régions ou d'autres pays. La marchandisation des connaissances des travailleurs fournit ainsi la matière première pour son expansion.

\section{VII}

Il y a maintenant une accumulation suffisante de telles connaissances pour impulser un développement énorme de la sous-traitance dans sa portée et son ampleur. Dans les cibles visées par les entreprises d'industrie des services (PSI) se trouve une large gamme de services, l'éducation et la santé étant considérées comme offrant les plus vastes domaines de croissance. ${ }^{41}$ Une prise particulièrement tentante est le British NHS (Service National de Santé), un des trois plus grands employeurs au monde (après l'armée rouge chinoise et les Chemins de fer nationaux indiens). ${ }^{42}$ Les conséquences de la crise financière en 2008 ont favorisé les conditions adéquates pour une telle croissance explosive. Le besoin de réduire les déficits des Etats (les dettes provoquées par le sauvetage des banques) légitime la réduction des coûts, la recherche d'« efficacité » et les économies d'échelle. Tout cela est étayé par une rhétorique sur la réduction du poids de l'Etat. Un nouveau modèle a été promu dans lequel la fonction du gouvernement n'est plus d'assurer des services mais de les proposer. Ce modèle a déjà été adopté avec enthousiasme par certains. Par exemple en octobre 2010, le Conseil du comté de Suffolk au Royaume-Uni a annoncé des plans pour devenir un "Conseil virtuel» et a sous-traité tous ses services, y compris les fonctions administratives, passant d'une main-d'œuvre de 27000 employés à environ $300 .{ }^{43}$ Le Barnet Council est déjà bien engagé dans cette voie avec sa politique "Future Shape » annoncée en 2008 qui s'est avérée avoir réalisé peu d'économies réelles, en partie à cause du coût élevé de la consultance, tout en conduisant à une baisse considérable de la qualité des services aussi bien qu'à des pertes d'emplois. ${ }^{44}$ Un exemple encore plus dramatique, même s'il est de moindre envergure, est celui de la toute petite ville de Maywood dans le 
comté de Los Angeles, qui depuis le $1^{\text {er }}$ juillet 2010 n'a plus aucun employé d'aucune sorte, tous ses services étant assurés par des entrepreneurs indépendants ou du personnel loué par la ville voisine de Bell. ${ }^{45}$

Au Royaume-Uni où le gouvernement de coalition fournit un exemple extrême de cette nouvelle façon de penser, les discours qui accompagnent ces développements ont été trompeurs, à gauche par la focalisation sur les « coupes budgétaires » qui insinue que le problème est seulement celui de la taille du budget dévolu aux services publics, et à droite par une rhétorique sur la "Big Society». Cette rhétorique ne parle pas de la remise directe de biens publics à des firmes multinationales pour les gérer (bien que cette option ne soit pas exclue) mais suggère qu'ils seront pris en charge par des volontaires. Le Livre vert Modernising Commissioning publié par le gouvernement britannique en 2010 cite l'engagement politique des Démocrates libéraux de «soutenir la création et l'expansion de mutuelles, de coopératives, d'entreprises sociales et caritatives et de rendre ces groupes en capacité d'avoir une plus grande implication dans le fonctionnement des services publics. » Il se poursuit en affirmant que « ces réformes sont fondamentales pour réaliser le Power Shift (la passation de pouvoir) qui a été confié à ce gouvernement pour faire passer le pouvoir du gouvernement central aux collectivités locales $\gg{ }^{46}$

Dans quelle mesure est-ce un simple écran de fumée ou un nouveau rôle offert aux ONG pour assurer des services, la question est discutable. À l'échelle mondiale, le rôle des ONG a considérablement changé ces dernières années. Non seulement nombre d'entre elles sont en partenariat à des degrés divers avec des firmes multinationales (par exemple, la Fondation Aga Khan assurant la formation d'infirmières en Tanzanie est financée par Johnson and Johnson), ${ }^{47}$ mais de nombreuses ONG sont entièrement financées par des multinationales. Par exemple, le New Citizen Life Center qui procure des services de santé et aide à la recherche d'un emploi les travailleurs migrants sans ressources dans le village de migrants Guijing à Pudong dans la périphérie de Shanghaï en Chine, est entièrement financé par Glaxo Smith Kline. Il sert à favoriser la promotion de produits de la firme aussi bien qu'à projeter une image positive de cette entreprise en particulier et du capitalisme en général. ${ }^{48} \mathrm{Il}$ semble probable qu'un mélange trouble des rôles de ce genre prendra place partout de plus en plus. Même si les services sont dirigés par des ONG, il n'apparaît pas clair à qui elles ont des comptes à rendre ni comment. À court terme, l'implication des organisations de volontaires peut adoucir et humaniser l'impact des changements ; à long terme, il semble probable que les firmes multinationales mettront fin à la prise de contrôle de toutes les opérations semblant être profitables, simplement en exploitant les économies d'échelle qu'elles peuvent réaliser. Il y a peu de doute que le principal impact de cette nouvelle approche sera le transfert massif de biens publics vers les firmes qui peuvent les utiliser pour générer des profits. Au cours du processus, la main-d'œuvre du secteur public sera subsumée en une masse plus grande de travailleurs interchangeables: disponibles, précaires et dépourvus de structures stables de représentation et de négociation collective, puisque les travailleurs seront de plus en plus employés par des entités ayant des positions intermédiaires dans la chaîne de valeur mondiale et changeante.

Cette situation n'est cependant pas sans contradictions. Au niveau de l'Etat, il existe des tensions entre, d'un côté, son rôle consistant à attirer et à contrôler le capital sur son territoire et, d'un autre côté, son rôle consistant à ouvrir de nouveaux champs d'expansion au capital. Au sein des élites nationales dirigeantes, il y a des tensions entre ceux qui veulent des gouvernements tout court* ayant un rôle plus restreint et ceux 
(représentant les entreprises qui profitent de la prestation de services publics) qui aimeraient voir une sphère publique élargie, à condition qu'elle soit ouverte pour faire des profits. Il est clair cependant, que si les travailleurs veulent récupérer des restitutions pour la classe ouvrière de la vague suivante d'accumulation (basée comme elle l'est sur l'expropriation de leur propre passé collectif d'efforts de redistribution), de nouvelles formes d'organisation seront requises: des formes d'organisation qui reconnaissent les intérêts communs d'un prolétariat mondial, ayant des employeurs mondialement organisés.

\section{NOTES}

1. On pourrait dire, peut-être plus précisément, que les valeurs d'usage non marchandes sont transformées en valeurs d'usage marchandes, leur donnant ainsi une valeur d'échange sur le marché.

2. Voir Claude Serfati, 'Transnational Organisations as Financial Groups', Work Organisation, Labour and Globalisation, 5(1), 2011, pour une discussion intéressante sur la convergence entre les sociétés transnationales financières et non financières.

3. UNCTAD World Investment Report, Genève, 2008, p. 3.

4. Ibid., p. 4.

5. Ibid., 2008, pp. XV-XVI.

6. Ibid., p. 4.

7. Par exemple, lorsqu'elle a été introduite à la fin des années 1990 dans l'industrie informatique pour effectuer des tâches lourdes de programmation, telles que la conversion des systèmes de comptabilité des compagnies européennes pour faire face à l'introduction de l'euro ou pour éviter les catastrophes annoncées pouvant être provoquées par le «bug du millénaire».

8. J'ai abondamment écrit ailleurs sur le long développement de cette nouvelle division mondiale du travail depuis les années 1970 dans, par exemple, U. Huws, The Making of a Cybertariat, New York: Monthly Review Press, 2003 ; U. Huws, 'Fixed, Footloose or Fractured : Work, Identity and the Spatial Division of Labour', Monthly Review, 57(10), 2006; U. Huws and J. Flecker, Asian Emergence: The World's Back Office? IES Report 419, Institute for Employment Studies, 2005.

9. Ce processus est décrit plus en détails dans U. Huws, 'The Restructuring of Global Value Chains and the Creation of a Cybertariat', in Christopher May, ed., Global Corporate Power : (Re)integrating Companies into International Political Economy, Boulder : Lynne Rienner Publishers, 2006, pp. 65-84.

10. D. Julius, Public Services Industry Review, London: Department for Business Enterprise and Regulatory Reform, 2008.

11. Comprenant les postes et télécommunications, les réseaux d'eau et d'énergie, les compagnies aériennes nationalisées, les banques nationalisées et le parc immobilier de l'Etat.

12. Données de l'OCDE, citées dans 'A Special Report on the Future of the State', The Economist, 19 mars 2011, p. 4.

13. Données du FMI, citées dans 'A Special Report', p. 5.

14. G. Esping-Andersen, The Three Worlds of Welfare Capitalism, New Jersey : Princeton University Press, 1990.

15. U. Huws, 'Move Over Brother', New Socialist, janvier 1985. 
16. Equal Opportunities Commission, The Gender Impact of CCT in Local Government, Manchester : Equal Opportunities Commission, 1995.

17. Ultérieurement, ce processus a culminé avec la directive sur les services en 2006 (2006/123) qui est entrée en vigueur le 28 décembre 2009 en renversant toutes les barrières nationales au sein de l'Union Européenne pour les sociétés souhaitant acquérir des services publics.

18. À nouveau, cela a créé le cadre pour une libéralisation allant plus loin au niveau du commerce international des services sous l'égide du GATS (Accord général sur le commerce et les services). Selon l'OMC même: «L'Uruguay Round n'était qu'un début. Le GATS demande plus de négociations, lesquelles ont commencé début 2000 et font maintenant partie du Programme de Doha pour le développement. L'objectif est de conduire le processus de libéralisation plus loin en augmentant le niveau des engagements dans les ordres du jour. " 'Understanding the WTO : the Agreements', disponible sur http://www.wto.org.

19. B. Jessop, The Future of the Capitalist State, Oxford : Polity Press, 2002.

20. La proportion de syndiqués est significativement plus élevée parmi les travailleurs du secteur public que parmi leurs homologues du secteur privé dans tous les pays européens sauf en Belgique. Voir V. Glassner, The Public Sector in the Crisis, Working Paper 2010.07, European Trade Union Institute, 2010, p. 15.

21. J'ai décrit ce processus de façon plus développée, par exemple dans Huws, The Making of a Cybertariat ; et dans Huws, 'The New Gold Rush', Work Organisation, Labour and Globalisation, 2(2), 2008.

22. Pour une dissection détaillée, voir S.Player et C. Leys, Confuse and Conceal: The NHS and Independant Sector Treatment Centres, London : Merlin Press, 2008.

23. Voir D. Whitfiefd, 'Marketisation of Legal Services', Legal Action, mars 2007.

24. C. Leys, Market-Driven Politics, London : Verso, 2003.

25. A. Hochschild, The Managed Heart : The Commercialization of Human Feeling, Berkeley : University of California Press, 1983.

26.

La question est parfois soulevée de savoir s'il y a une amélioration dans la qualité des services qui ont été standardisés, marchandisés et sous-traités. Si cela est le cas, on sous-entend alors que les avantages pour les usagers peuvent l'emporter sur les désavantages pour les travailleurs. En fait, il est extrêmement difficile de se livrer à ce genre de comparaisons pour de nombreuses raisons. Premièrement, une restructuration est souvent introduite dans des situations où les services sont déjà détériorés à cause des coupes budgétaires. Deuxièmement, les processus de changement associés à la marchandisation sont difficiles à comparer terme à terme. Troisièmement, la focalisation obsessionnelle sur les indices quantitatifs qui sont un élément essentiel étayant la marchandisation rend invisible de nombreux changements qualitatifs qui peuvent être vécus négativement par les usagers. Quoi qu'il en soit, il y a une masse de travaux de recherche qui laissent entendre qu'il y a une détérioration.

(voir par exemple C. Leys et A. Pollock, NHS plc: The Privatisation of our Health Care, London : Verso, 2004 ; Player et Leys, Confuse and Conceal ; D. Whitfield, Global Auction of Public Assets, London: Spokesman,

2009). 
Ce n'est peut-être pas un hasard si John Hutton, secrétaire d'Etat aux affaires, aux entreprises et à la réforme réglementaire dans le gouvernement travailliste en 2008, a même arrêté de soutenir que le principal avantage de la sous-traitance était d'apporter des gains d'efficacité. La Public Services Industry (PSI) devrait être encouragée, a-t-il dit parce que « Il y a un potentiel d'exportation significatif avec la croissance de cette industrie. Aider et encourager les firmes britanniques à saisir au maximum ces opportunités générera des bénéfices substantiels, pas seulement pour les firmes britanniques mais aussi pour l'économie britannique. La revue (PSI Review) conclut que la meilleure manière qu'a le gouvernement de soutenir la PSI à l'étranger est de maintenir un encadrement compétitif pour les services publics qui puisse favoriser une PSI dynamique et florissante au Royaume-Uni ».

Voir l'Executive Summary introduction à Julius, Public Services Industry, p. v.

27.

L'Organisation internationale de normalisation qui a 2700 comités techniques, sous-comités et groupes de travail, a fixé des normes techniques internationales pour une vaste gamme de processus industriels différents. L'existence de ces normes signifie qu'il est possible de commercer, ou de sous-traiter, avec une entreprise certifiée ISO, en étant confiant que les résultats produits seront prévisibles et normalisés, écartant ainsi le besoin d'un contrôle détaillé ; c'est, par exemple, tout à fait de la même façon que les normes électriques permettent de brancher un appareil à une prise standard, en étant sûr qu'il fonctionnera correctement. 28. ISO Survey, 2009, disponible sur http://www.iso.org .

29. Dans ce cas: http://www.certicationskit.com/cisco-certification/cisco-certification-salarystatistics.

30. B. Caraway, 'Online Labour Markets : An Enquiry into oDesk Providers', Work Organisation, Labour and Globalisation, 4(2), 2010, pp. 111-125.

31. Disponible sur http://www.training.com

32.

J'ai écrit de façon plus approfondie sur le changement des identités professionnelles des travailleurs informaticiens dans le contexte de la mondialisation dans U. Huws, 'New Forms of Work ; New Occupational Identities', dans N. Pupo and M. Thomas, eds., Interrogating the 'New Economy' : Restructuring Work in the 21st Century, Peterborough, Ontario : Broadview Press, 2010.

33. Voir C. Dixon, 'The Reformatting of State Control in Vietnam', Work Organisation, Labour and Globalisation, 2(2), 2008, pp. 101-118.

34. Disponible sur http://www.serco.com.

35. C. Leys et S. Player, The Plot Against the NHS, London : Merlin Press, 2011.

36.

Pour une description détaillée de l'impact de la « callcenterisation » sur les compétences des travailleurs du système des impôts danois, voir Bramming, Sørensen et Hasle, 'In Spite of Everything: Professionalism as Mass Customised Bureaucratic Production in a Danish Government Call Center', Work Organisation, Labour and Globalisation, 3(1), 2009, pp. 114-130.

37. Voir P. Meil, P. Tengblad et P. Docherty, Value Chain Restructuring and Industrial Relations - The Role of Workplace Representation in Changing Conditions of Employment and Work, WORKS Project, Higher Institute of Labour Studies, K. U Leuven, Leuven : HIVA, 2009.

38. Cette étude de cas est décrite plus en détails dans S. Dahlmann, 'The End of the Road: No More Walking in Dead Men's Shoes: IT Professionals' Experience of being Outsourced to the Private Sector', Work Organisation, Labour and Globalisation, 2(2), 2008, pp. 148-161.

39. H. Reed, The Shrinking State: Why the Rush to Outsource Threatens our Public Services, London : A report for Unite by Landman Economics, 2011, p. 13.

40. Ibid., p. 18. 
41. Julius, Public Services Industry Review.

42. S. Lister, 'NHS is World's Biggest Employer after Indian Rail and Chinese Army', The Times Online, 20 mars 2004.

43. A. Bawden, 'Suffolk Council Plans to Outsource Virtually all Services', Guardian.co.uk, 22 septembre 2010.

44. D. Whitfield, Analysis of Development and Regulatory Services Business Case, London: European Services Strategy Unit, 2011.

45. 'There Goes Everybody', The Economist, 8 juillet 2010.

46. Cabinet Office, Modernising Commissioning: Inceasing the Role of Charities, Social Enterprises, Mutuals and Co-operatives in Public Service Delivery, Cabinet Office Green Paper, London, 2010, p. 5, les italiques étant dans l'original.

47. Aga Khan Development Network, 'Upgrading Nursing Studies : Strengthening the Health-Care System in Tanzania', octobre 2007, disponible sur http://www.akdn.org.

48. B. Neilson, 'Guijing Migrant Village’, Transit Labour, 2 décembre 2010, pp. 33-35. 